\title{
Left Atrial Appendage Closure in Atrial Fibrillation: A World without Anticoagulation?
}

\author{
Tahmeed Contractor ${ }^{1}$ and Atul Khasnis ${ }^{2}$ \\ ${ }^{1}$ Department of Medicine, Michigan State University, B301 Clinical Center, East Lansing, MI 48824, USA \\ ${ }^{2}$ Department of Rheumatologic and Immunologic Diseases, Center for Vasculitis Care and Research, Cleveland Clinic, \\ Cleveland, OH 44195, USA
}

Correspondence should be addressed to Tahmeed Contractor, tahmeedcontractor@gmail.com

Received 20 December 2010; Accepted 1 February 2011

Academic Editor: Adrian Baranchuk

Copyright (C) 2011 T. Contractor and A. Khasnis. This is an open access article distributed under the Creative Commons Attribution License, which permits unrestricted use, distribution, and reproduction in any medium, provided the original work is properly cited.

\begin{abstract}
Atrial Fibrillation (AF) is a common arrhythmia with an incidence that is as high as $10 \%$ in the elderly population. Given the large proportion of strokes caused by $\mathrm{AF}$ as well as the associated morbidity and mortality, reducing stroke burden is the most important part of AF management. While warfarin significantly reduces the risk of AF-related stroke, perceived bleeding risks and compliance limit its widespread use in the high-risk AF population. The left atrial appendage is believed to be the "culprit" for thrombogenesis in nonvalvular AF and is a new therapeutic target for stroke prevention. The purpose of this review is to explore the evolving field of percutaneous LAA occlusion. After briefly highlighting the risk of stroke with AF, problems with warfarin, and the role of the LAA in clot formation, this article discusses the feasibility and efficacy of various devices which have been developed for percutaneous LAA occlusion.
\end{abstract}

\section{Introduction}

Atrial fibrillation $(\mathrm{AF})$ is the most common sustained arrhythmia encountered in clinical practice. It has an estimated prevalence of $1 \%$ in the adult population translating into greater than two million cases in the United States [1]. The projected number of individuals with AF in the United States of America is expected to be approximately 10 million by 2050 [2]. With better methods of diagnosing AF, especially paroxysmal AF, as well as increasing physician awareness, the actual burden may be higher than expected. AF is fraught with the serious complication of thromboembolism. Although anticoagulation is effective, patients and physicians both seek alternative means to avert the risk of stroke from thromboembolism due the need for monitoring and bleeding complications as well as the potential for drug interactions. Left atrial appendage (LAA) closure devices have become an attractive option for this purpose. In this review, we will summarize the available literature and evidence for use of percutaneous left atrial appendage closure as an alternative to chronic anticoagulation.

\section{Stroke Risk in Atrial Fibrillation}

AF increases the risk of stroke by 4 to 5 folds in nonrheumatic patients [3] and 17 folds in the setting of rheumatic mitral stenosis [4]. It is responsible for $10 \%$ of all ischemic strokes and half of all cardioembolic strokes [5]. The impact of AF as a risk factor for stroke increases with age. While the annual incidence of stroke due to atrial fibrillation is $1.5 \%$ in patients aged 50-59 years, almost a quarter of the strokes in patients aged 80 to 89 years are secondary to AF [3]. Perceived adverse effects related to anticoagulation also increase with age, which may result in a paradoxical underuse in a population at highest risk for stroke [6].

Paroxysmal AF (PAF), which accounts for $25 \%$ of AF, has the same risk of stroke as permanent/persistent AF [7]. In a recent study, up to $23 \%$ patients with stroke/TIA of unknown etiology (cryptogenic stroke) were found to have PAF on subsequent monitoring [8]. Given that cryptogenic stroke accounts for $36 \%$ of all strokes [9], the stroke burden attributed to PAF has been hitherto significantly underestimated. With better methods of diagnosing PAF in patients 
with cryptogenic stroke, such as transtelephonic EKG monitoring [10] and mobile cardiac outpatient telemetry [8], the number of patients potentially requiring anticoagulation is expected to increase in the future.

Apart from a sheer increase in numbers, AF-related strokes also tend to be more severe when compared to other causes of thromboembolism, likely due to the larger size of thrombi $[11,12]$. Consequently, there is a significant impact on the quality of life as well as longevity with an estimated 28-day mortality of $20 \%$ post-AF-related stroke [13]. Thus, reducing the stroke burden is a sine qua non of AF management.

\section{Underuse of Anticoagulation in AF}

Warfarin dramatically decreases the risk of stroke in patients with valvular as well as nonvalvular AF. In a meta-analysis of randomized controlled trials, warfarin resulted in a $64 \%$ relative risk reduction for stroke when compared to placebo [14]. Another meta-analysis revealed that warfarin is almost three times more effective than antiplatelet agents in preventing strokes (relative risk reduction $64 \%$ for warfarin versus $20 \%$ for antiplatelet agents) [15].

However, several studies have reported underuse of warfarin in eligible patients with AF. In a large cross-sectional study, $45 \%$ patients with moderate to high-risk AF did not receive warfarin [16]. Similarly, a recent cohort study of Medicare beneficiaries with atrial fibrillation revealed that only two thirds of ideal anticoagulation candidates were prescribed warfarin, with significant differences in use, monitoring, and effectiveness of warfarin among different ethnic groups [17]. Given the high incidence of atrial fibrillation and associated risk factors for stroke, a small percentage of underuse could translate into a huge hurdle to stroke prevention in the population.

Table 1 outlines important reasons for warfarin underutilization. Fear of major bleeding, especially hemorrhagic strokes and gastrointestinal bleeding, is a major reason for this underuse $[18,19]$. This fear is not without reason; a meta-analysis revealed that the risk of intracranial hemorrhage is doubled with warfarin when compared with aspirin [15]. Anticoagulants ranked first in 2003 and 2004 in the number of deaths from drugs causing "adverse effects in therapeutic use" [20]. The perceived risk is greater in elderly patients with AF [21], and "fall risk" is estimated in rather ambiguous ways in this population. Inconvenience of INR monitoring for rural populations, drug, and dietary interactions as well as compliance are other limiting factors [22]. For unclear reasons, warfarin is also underused in females compared to males [23].

Novel anticoagulants, such as the thrombin inhibitors (dabigatran) and the factor Xa inhibitors (rivaroxaban) have a wider therapeutic window. There is minimal dietary and drug interference with these agents and INR monitoring is not required. While these may decrease many of the concerns associated with warfarin, they do not address issues related to bleeding and compliance [24]. A case in point is that of dabigatran, which is superior to warfarin in preventing
TABLE 1: Causes of warfarin underutilization.

(1) Risk of bleeding (perceived or real)

(a) Intracranial hemorrhage

(b) Gastrointestinal bleeding

(c) Other major bleeds (retroperitoneal, hematuria, etc.)

(2) Perceived Fall Risk (and associated bleeding risk)

(3) Difficulty with INR monitoring

(a) Geographical barriers

(b) Lack of insurance/primary care physician

(4) Non-compliance

(5) Patient preference

(6) Physician unawareness (such as need in paroxysmal $\mathrm{AF}^{*}$ )

(7) Pharmacokinetic interference

(a) Drug interaction

(b) Dietary interference

(8) Ethnic and gender disparities

*AF: atrial fibrillation.

strokes but has a similar risk of major bleeding at the currently approved dose [25]. Similarly, there was no difference in major bleeding event rates between rivuroxaban and warfarin groups in the recent ROCKET-AF trial [26]. Also, cost-effectiveness and efficacy of these novel agents in real world setting remain to be seen.

\section{Left Atrial Appendage: The Culprit?}

The left atrial appendage is an embryological remnant that functions during conditions of volume overload as a reservoir and mediator of adaptive responses to decrease circulating blood volume [27]. Compared to the right atrial appendage, the LAA is anatomically prone to stasis by virtue of having a long, tubular structure as well as a narrow junction with the atrium [28]. Multiple studies have found a predilection for thrombus to form within the LAA in patients with mitral valve disease (irrespective of underlying rhythm) and nonvalvular AF.

The hematological and endocardial substrate for thrombogenesis in the LAA is yet to be elucidated, but the structural basis has been defined. All known variables associated with thrombus formation, including LAA size and flow pattern, are altered due to AF-associated LAA dysfunction [27]. Postmortem LAA cast analysis from patients with AF revealed that its size was much larger in patients with AF [29]. A specific type of LAA flow pattern in patients with AF (Type III indicating no identifiable flow waves with AF) was associated with a higher incidence of LAA thrombus [30]. These represent the macroscopic basis of LAA thrombus formation in the setting of AF.

In contrast to patients with valvular AF, it is hypothesized that the LAA may be the major source for emboli in nonvalvular AF. This is based on an analysis of 23 studies utilizing echocardiographic, operative, and postmortem evaluations of the left atrium and LAA in patients with AF (Table 2). The percentage of thrombi found in the LAA was significantly higher in patients with nonvalvular AF than in valvular 
TABLE 2: Left atrial appendage as a source of thrombi in nonatrial fibrillation.

(i) Hypothesis is based on a landmark study by Blackshear and Odell [31]

(ii) Findings from studies in settings of operation, autopsy, or transesophageal echocardiography were combined

(iii) 23 studies including close to 5,000 subjects with rheumatic or nonrheumatic $\mathrm{AF}^{*}$ were reviewed

(iv) Thrombi presented in the appendage but extending into the atrium was classified as a LA** thrombus

(v) While $57 \%$ of atrial thrombi in rheumatic AF occurred in the appendage, $91 \%$ of left atrial thrombi were located in the atrial appendage in nonrheumatic $\operatorname{AF}(P<.0001)$

(vi) Many have pointed out that merely finding a thrombus in the LAA does not prove that it is the source of cardio-emboli in AF-related ischemic stroke [32]

(vii) This study set the stage to investigate the benefit of LAA occlusion in reducing nonrheumatic AF-related stroke burden

${ }^{*}$ AF: atrial fibrillation; ${ }^{*}$ LAA: left atrial appendage.

AF (91\% versus 57\%; $P<.001$ ) [31]. This data, however, does not confirm that the LAA is the "source" of emboli in AF-related ischemic stroke, and many have questioned this theory [32]. Nevertheless, many surgical as well as noninvasive methods to occlude the LAA have been assessed to reduce stroke burden and confirm this hypothesis.

\section{Methods of LAA Occlusion}

5.1. Surgical. Several studies have assessed the feasibility, safety, and efficacy of surgical LAA occlusion [33, 34]. Given its invasive nature, surgical LAA occlusion is only performed in patients requiring other cardiac surgery such as mitral valve surgery and maze procedures. The addition of this simple, quick procedure to the aforementioned surgeries can reduce stroke burden in many patients with established AF or at high risk for AF. However, its efficacy for occluding the LAA and preventing thromboembolic events has been questioned, with a study demonstrating that surgical LAA occlusion is frequently incomplete [35]. Continuous advancements in open surgical LAA occlusion technique are being made with better methods for intraoperative confirmation of success (TEE), and outcomes are expected to improve in the future.

5.2. Nonsurgical. There are several percutaneous devices, which have been used for LAA occlusion. Of these, only the WATCHMAN device is FDA approved. Table 3 summarizes studies, which have analyzed the feasibility and efficacy of different devices.

\section{The Amplatzer Septal Occluder (AGA Medical Corp., Golden Valley, Minn)}

The Amplatzer septal occluder, which was originally used for patent foramen ovale or atrial septal defect closure, was tested for LAA closure in a preliminary analysis of 16 patients across four centers [36]. There was a single event of asymptomatic device embolization due to inappropriate size; no other complications or thromboembolic episodes were reported in a short followup period of four months. However, no further trials were conducted with this system, which paved the way for the LAA-specific devices. The Amplatzer Cardiac Plug is another device by the same manufacturer which is currently being compared with warfarin in the Amplatzer Cardiac Plug trial.

\section{The PLAATO System (eV3, Plymouth, Minn)}

The PLAATO implant consists of a self-expanding nitinol cage coated with a special expanded polytetrafluoroethylene (ePTFE) membrane to occlude blood flow into the orifice while allowing tissue incorporation. The ePTFE membrane ensures benign healing [42], resulting in endothelialization of the implant in 2 to 3 months. A custom 14Fr transseptal sheath is used to deliver the implant into the LAA, and small anchors along the struts of the implant hold it in position.

After initial animal studies confirmed safe LAA occlusion with this system [43], a feasibility study was conducted in Europe in 15 patients with chronic, nonrheumatic AF and contraindications to warfarin therapy [37]. All patients were at high risk for thromboembolism based on CHADS2 criteria [44] or spontaneous echo contrast in the LAA on TEE. The device was successfully implanted in all patients with an average implant time of 90 minutes. Periprocedural hemopericardium delayed successful implantation in one patient by a month, and the device needed to be exchanged due to inappropriate size in 4 patients. A one month followup showed stable implant position, and there were no reported late complications or embolic events. An addendum to this study reported successful implantation in 16 additional patients (with another case of hemopericardium).

Subsequently, the larger PLAATO multicenter study reported results from multiple sites in North America and Europe with a followup period of up to 17 months [38]. Patients with nonrheumatic AF of at least three months duration with contraindications to warfarin and high risk for thromboembolic events were included. The latter was based on CHADS2 criteria, presence of coronary artery disease, or echographic criteria (moderate to dense spontaneous echocardiographic contrast or blood flow velocity $<20 \mathrm{~cm} / \mathrm{s}$ within the LAA), thus expanding inclusion criteria from the previous feasibility study. Percutaneous LAA occlusion was successful in 108 of 111 patients, with an average procedure time of 68 minutes. There were two reported major adverse events in a single patient within the first month. A total of 5 patients $(4.5 \%)$ experienced hemopericardium during the entire followup; four of these five had no long-term sequelae. Based on a mean CHADS2 score of 2.5, the estimated stroke risk in the population was $6.5 \%$. This was reduced to almost a third (2.2\%/year) with the PLAATO system for strokes alone; TIA was not included.

The largest feasibility study for the PLAATO system was conducted in North-America and utilized a prospective, nonrandomized study design enrolling 64 patients across 


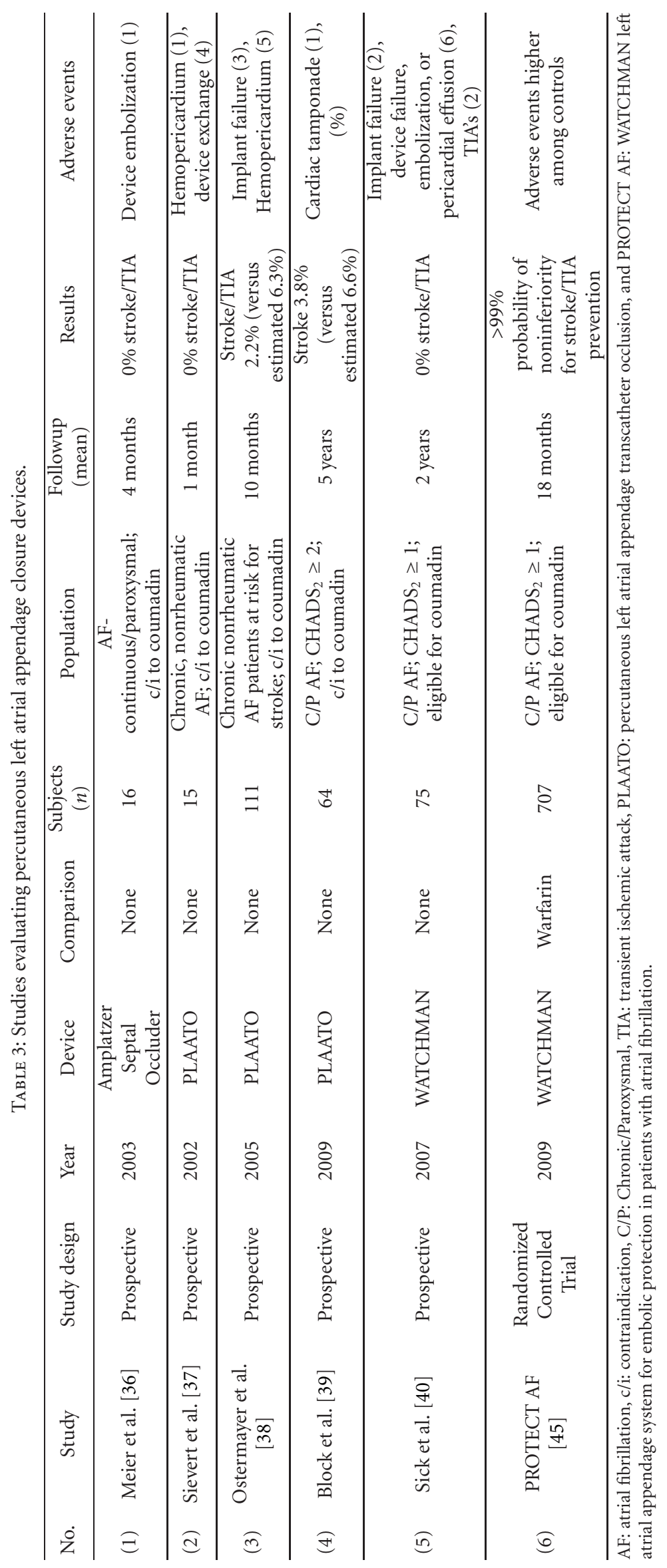


10 centers [39]. The inclusion criteria for this study were broader than the previous, smaller feasibility studies. Due to the similar risk for thromboembolic events, patients with either permanent or paroxysmal AF were selected for inclusion. Risk for developing thromboembolic events was defined by a CHADS2 score $\geq 2$ or the presence of any of the multiple high-risk echocardiographic findings. More than $98 \%$ of the patients met the primary end point of no major adverse events (i.e., stroke, cardiac, or neurological death, MI or requirement of surgery related to the PLAATO procedure). In the five-year followup, there was only one adverse event (cardiac tamponade) attributed to the procedure, indicating an improved safety profile with time and experience (the learning curve effect). There were no other procedure-related adverse events, device failures, or malfunctions throughout the study period. The estimated yearly combined rate of stroke/TIA was $6.6 \%$, calculated from the mean CHADS2 score of 2.6 in the study population. This was reduced to a rate of a $3.8 \%$ /year with the PLAATO system. Despite the encouraging results, there were no subsequent studies comparing the device to warfarin therapy.

\section{WATCHMAN Device (Atritech Inc., Plymouth, Minn)}

Similar to the PLAATO implant, the WATCHMAN LAA closure device consists of a self-expanding nitinol frame [41]. It also has fixation barbs; however, the permeable polyester fabric only covers the surface exposed to the left atrium. It is available in five different sizes ranging from a diameter of 21 to $33 \mathrm{~mm}$. The implant is preloaded within a catheter and requires an access sheath for delivery into the LAA.

A preliminary feasibility study tested this device in 75 patients across multiple centers in Europe and North America. Subjects with chronic or paroxysmal nonvalvular AF eligible for warfarin therapy and a CHADS2 score $\geq 1$ were included [40], thus having broader inclusion criteria than feasibility studies for PLAATO. After implantation, patients were discharged on warfarin which was discontinued at 45 days if LAA remained successfully sealed by echocardiographic criteria. The initial 16 patients received the first generation device. The device was subsequently redesigned and implantation of a second-generation device was attempted in 59 patients. Adverse effects were much lesser with second- than first-generation device, including core wire failure (0/59 versus $2 / 16)$, device embolization (0/59 versus $2 / 16)$, and internal bleeding (0/59 versus $1 / 16)$. Though minor pericardial effusions were more common with the second-generation device (3/59 versus 0/16 with first generation), there was one case of pericardial effusion requiring treatment in each group. Based on the CHADS2 score, the expected annual stroke risk of $1.9 \%$ was reduced to $0 \%$ with this device over a followup period of two years.

The PROTECT AF (WATCHMAN Left Atrial Appendage System for Embolic Protection in Patients with Atrial Fibrillation) is the first study that directly compared a LAA occlusion device head-to-head with warfarin therapy [45]. It was primarily designed to assess noninferiority of the WATCHMAN device against warfarin. This prospective randomized control trial used inclusion criteria similar to the initial feasibility study, choosing patients with paroxysmal, persistent or permanent nonvalvular atrial fibrillation, and a CHADS2 score of $\geq 1$. A computer-generated sequence randomized selected patients $(n=707)$ to the device $(n=463)$ and warfarin therapy $(n=244)$ in a $2: 1$ ratio. Those randomized to intervention subsequently discontinued warfarin after transesophageal echocardiographic (TEE) demonstration of complete LAA closure or a residual jet $<5 \mathrm{~mm}$ in width. Following this, dual antiplatelet therapy (aspirin and clopidogrel) was prescribed until the end of a 6month followup period. This was in contrast to the PLAATO recipients, who only received dual antiplatelet therapy for four to six weeks without any warfarin therapy. The device was successfully implanted in $88 \%$ of those assigned to the intervention group, of which $92 \%$ could stop taking warfarin at 6 months. The primary efficacy event rate for occurrence of stroke (ischemic or hemorrhagic), cardiovascular or unexplained death, or systemic embolism was $3 / 100$ patientyears in the intervention group and 4.9/100 patient-years in the control group (rate ratio [RR] 0.62, 95\% CI 0.351.25 ) with a greater than $99 \%$ probability of noninferiority. Though the primary safety event rate for occurrence of hemorrhage or procedure related complications was greater in the intervention group of the "intention to treat" population (7.5 versus 4.4 per 100 patient-years; RR 1.69 (95\% CI 1.01$3.19)$ ), the rate was higher in the control group amongst those successfully treated with device or warfarin (1.5 versus 4.4 per 100 patient-years; RR 0.35 (95\% CI 0.15-0.80)).

There were several concerns about the trial design, such as using a noninferiority hypothesis to compare two different modalities of treatment, that is, a drug and a device. The risk of hemorrhagic stroke in the warfarin group (1.6 per 100 patient-years) was much higher than that reported in previous trials $(0.5$ per 100 patient-years in a meta-analysis of 6 randomized clinical trials [46]) and this may have influenced the noninferiority results for primary efficacy. Also, the rate of serious pericardial effusion requiring drainage was fairly high in the device group $(4.8 \%$ versus $0 \%$ ). The FDA has recommended longer followup studies with this device, and another trial is being planned by investigators.

\section{Future Perspective}

Percutaneous LAA occlusion devices may represent the "holy grail" in reducing nonvalvular AF-related stroke burden. Researchers have been constantly looking for easier methods for this, and a single procedure with short-term anticoagulation is more feasible than life-long anticoagulation and associated bleeding risks. Oral anticoagulants may become "alternative" agents in nonvalvular AF subjects who are not suitable for device implantation.

However, there are many questions that remain unanswered. Though only the WATCHMAN device is under consideration for FDA approval, results from feasibility studies of PLAATO are encouraging. A trial with the 
Amplatzer Cardiac Plug device is also underway. Long-term studies with these devices and head-to-head comparisons may be necessary before deciding the "best" device. Different trials have used varied regimens for periprocedural antiplatelet/anticoagulant therapy and contrasting these protocols is imperative. If postprocedural warfarin therapy is needed, populations at high-risk or ineligible for warfarin therapy may also be unsuitable for device implantation. This will leave a huge proportion of the AF population at highrisk for stroke without any therapeutic modality. Despite the benefit of LAA occlusion, procedure-related adverse events such as pericardial effusion and device embolization remain concerning. Once this procedure gains popularity, longer followup studies in real-world settings will help assess the actual risk of adverse events. The role of this device in the face of newer, safer, and more efficacious oral anticoagulants, such as dabigatran and rivoroxaban, also remains to be seen. A recent indirect comparison of data from the PROTECT-AF and RE-LY trial revealed that the Watchman device would fail to meet noninferiority when compared to dabigatran [47]. Prospective, head-to-head trials are required to address this question.

\section{Conclusion}

While warfarin has significantly reduced stroke risk in patients with AF, it is still underused secondary to perceived risk for hemorrhagic complications and drug interactions. The LAA is considered to play a major role in thrombogenesis in patients with nonvalvular AF and is a new target for stroke prevention. The FDA has withheld the approval of WATCHMAN device, and better designed trials with a longer followup are necessary to evaluate this method in comparison to warfarin and novel oral anticoagulants.

\section{References}

[1] A. S. Go, E. M. Hylek, K. A. Phillips et al., "Prevalence of diagnosed atrial fibrillation in adults: National implications for rhythm management and stroke prevention: the anticoagulation and risk factors in atrial fibrillation (ATRIA) study," Journal of the American Medical Association, vol. 285, no. 18, pp. 2370-2375, 2001.

[2] Y. Miyasaka, M. E. Barnes, B. J. Gersh et al., "Secular trends in incidence of atrial fibrillation in Olmsted County, Minnesota, 1980 to 2000, and implications on the projections for future prevalence," Circulation, vol. 114, no. 2, pp. 119-125, 2006.

[3] P. A. Wolf, R. D. Abbott, and W. B. Kannel, "Atrial fibrillation as an independent risk factor for stroke: the Framingham Study," Stroke, vol. 22, no. 8, pp. 983-988, 1991.

[4] P. A. Wolf, T. R. Dawber, H. E. Thomas, and W. B. Kannel, "Epidemiologic assessment of chronic atrial fibrillation and risk of stroke: the Framingham study," Neurology, vol. 28, no. 10, pp. 973-977, 1978.

[5] R. G. Hart, L. A. Pearce, R. M. Rothbart, J. H. McAnulty, R. W. Asinger, and J. L. Halperin, "Stroke with intermittent atrial fibrillation: incidence and predictors during aspirin therapy," Journal of the American College of Cardiology, vol. 35, no. 1, pp. 183-187, 2000.
[6] R. G. Hart, "Warfarin in atrial fibrillation: underused in the elderly, often inappropriately used in the young," Heart, vol. 82, no. 5, pp. 539-540, 1999.

[7] S. H. Hohnloser, D. Pajitnev, J. Pogue et al., "Incidence of stroke in paroxysmal versus sustained atrial fibrillation in patients taking oral anticoagulation or combined antiplatelet therapy: an ACTIVE W Substudy," Journal of the American College of Cardiology, vol. 50, no. 22, pp. 2156-2161, 2007.

[8] A. H. Tayal, M. Tian, K. M. Kelly et al., "Atrial fibrillation detected by mobile cardiac outpatient telemetry in cryptogenic TIA or stroke," Neurology, vol. 71, no. 21, pp. 1696-1701, 2008.

[9] G. W. Petty, R. D. Brown Jr., J. P. Whisnant, J. D. Sicks, W. M. O'Fallon, and D. O. Wiebers, "Ischemic stroke subtypes: a population-based study of incidence and risk factors," Stroke, vol. 30, no. 12, pp. 2513-2516, 1999.

[10] N. Gaillard, S. Deltour, B. Vilotijevic et al., "Detection of paroxysmal atrial fibrillation with transtelephonic EKG in TIA or stroke patients," Neurology, vol. 74, no. 21, pp. 1666-1670, 2010.

[11] M. J. G. Harrison and J. Marshall, "Atrial fibrillation, TIAs and completed strokes,” Stroke, vol. 15, no. 3, pp. 441-442, 1984.

[12] D. C. Anderson, L. J. Kappelle, M. Eliasziw, V. L. Babikian, L. A. Pearce, and H. J. M. Barnett, "Occurrence of hemispheric and retinal ischemia in atrial fibrillation compared with carotid stenosis," Stroke, vol. 33, no. 8, pp. 1963-1967, 2002.

[13] M. M. Kaarisalo, P. Immonen-Räihä, R. J. Marttila et al., "Atrial fibrillation and stroke: mortality and causes of death after the first acute ischemic stroke," Stroke, vol. 28, no. 2, pp. 311-315, 1997.

[14] J. Morley, R. Marinchak, S. J. Rials, and P. R. Kowey, "Atrial fibrillation, anticoagulation, and stroke," American Journal of Cardiology, vol. 77, no. 3, pp. 38A-44A, 1996.

[15] R. G. Hart, L. A. Pearce, and M. I. Aguilar, "Meta-analysis: antithrombotic therapy to prevent stroke in patients who have nonvalvular atrial fibrillation," Annals of Internal Medicine, vol. 146, no. 12, pp. 857-867, 2007.

[16] A. L. Waldo, R. C. Becker, V. F. Tapson, and K. J. Colgan, "Hospitalized patients with atrial fibrillation and a high risk of stroke are not being provided with adequate anticoagulation," Journal of the American College of Cardiology, vol. 46, no. 9, pp. 1729-1736, 2005.

[17] E. Birman-Deych, M. J. Radford, D. S. Nilasena, and B. F. Gage, "Use and effectiveness of warfarin in medicare beneficiaries with atrial fibrillation," Stroke, vol. 37, no. 4, pp. 1070-1074, 2006.

[18] R. J. Beyth, M. R. Antani, K. E. Covinsky et al., "Why isn't warfarin prescribed to patients with nonrheumatic atrial fibrillation?" Journal of General Internal Medicine, vol. 11, no. 12, pp. 721-728, 1996.

[19] B. F. Gage, M. Boechler, A. L. Doggette et al., "Adverse outcomes and predictors of underuse of antithrombotic therapy in medicare beneficiaries with chronic atrial fibrillation," Stroke, vol. 31, no. 4, pp. 822-827, 2000.

[20] D. K. Wysowski, P. Nourjah, and L. Swartz, "Bleeding complications with warfarin use: a prevalent adverse effect resulting in regulatory action," Archives of Internal Medicine, vol. 167, no. 13, pp. 1414-1419, 2007.

[21] M. Kutner, G. Nixon, and F. Silverstone, "Physicians' attitudes toward oral anticoagulants and antiplatelet agents for stroke prevention in elderly patients with atrial fibrillation," Archives of Internal Medicine, vol. 151, no. 10, pp. 1950-1953, 1991. 
[22] J. A. O’Hare, N. Ul-Iman, and M. Geoghegan, "Non-anticoagulation in atrial fibrillation," Irish Journal of Medical Science, vol. 163, no. 10, pp. 448-450, 1994.

[23] N. L. Smith, B. M. Psaty, C. D. Furberg et al., “Temporal trends in the use of anticoagulants among older adults with atrial fibrillation," Archives of Internal Medicine, vol. 159, no. 14, pp. 1574-1578, 1999.

[24] S. Haas, "New anticoagulants—-towards the development of an "ideal" anticoagulant," Vasa, vol. 38, no. 1, pp. 13-29, 2009.

[25] S. J. Connolly, M. D. Ezekowitz, S. Yusuf et al., "Dabigatran versus warfarin in patients with atrial fibrillation," New England Journal of Medicine, vol. 361, no. 12, pp. 1139-1151, 2009.

[26] I. Ehrens, G. Y. H. Lip, and K. Peters, "What do the RELY, AVERROES and ROCKET-AF trials tell us for stroke prevention in atrial fibrillation?" Thrombosis and Haemostasis, vol. 105, no. 4, 2011.

[27] N. M. Al-Saady, O. A. Obel, and A. J. Camm, "Left atrial appendage: structure, function, and role in thromboembolism," Heart, vol. 82, no. 5, pp. 547-554, 1999.

[28] T. W. Sadler, "Cardiovascular system," in Langman's Medical Embryology, J. Langman, Ed., pp. 179-227, Williams and Wilkins, Baltimore, Md, USA, 6th edition, 1990.

[29] G. Ernst, C. Stöllberger, F. Abzieher et al., "Morphology of the left atrial appendage," Anatomical Record, vol. 242, no. 4, pp. 553-561, 1995.

[30] M. A. García-Fernandez, E. G. Torrecilla, D. S. Román et al., "Left atrial appendage Doppler flow patterns: implications on thrombus formation," American Heart Journal, vol. 124, no. 4, pp. 955-961, 1992.

[31] J. L. Blackshear and J. A. Odell, "Appendage obliteration to reduce stroke in cardiac surgical patients with atrial fibrillation," Annals of Thoracic Surgery, vol. 61, no. 2, pp. 755-759, 1996.

[32] R. P. Whitlock, J. S. Healey, and S. J. Connolly, "Left atrial appendage occlusion does not eliminate the need for warfarin," Circulation, vol. 120, no. 19, pp. 1927-1932, 2009.

[33] J. S. Healey, E. Crystal, A. Lamy et al., "Left Atrial Appendage Occlusion Study (LAAOS): results of a randomized controlled pilot study of left atrial appendage occlusion during coronary bypass surgery in patients at risk for stroke," American Heart Journal, vol. 150, no. 2, pp. 288-293, 2005.

[34] M. A. García-Fernández, E. Pérez-David, J. Quiles et al., "Role of left atrial appendage obliteration in stroke reduction in patients with mitral valve prosthesis: a transesophageal echocardiographic study," Journal of the American College of Cardiology, vol. 42, no. 7, pp. 1253-1258, 2003.

[35] E. S. Katz, T. Tsiamtsiouris, R. M. Applebaum, A. Schwartzbard, P. A. Tunick, and I. Kronzon, "Surgical left atrial appendage ligation is frequently incomplete: a transesophageal echocardiographic study," Journal of the American College of Cardiology, vol. 36, no. 2, pp. 468-471, 2000.

[36] B. Meier, I. Palacios, S. Windecker et al., "Transcatheter left atrial appendage occlusion with Amplatzer devices to obviate anticoagulation in patients with atrial fibrillation," Catheterization and Cardiovascular Interventions, vol. 60, no. 3, pp. 417-422, 2003.

[37] H. Sievert, M. D. Lesh, T. Trepels et al., "Percutaneous left atrial appendage transcatheter occlusion to prevent stroke in high-risk patients with atrial fibrillation: early clinical experience," Circulation, vol. 105, no. 16, pp. 1887-1889, 2002.
[38] S. H. Ostermayer, M. Reisman, P. H. Kramer et al., "Percutaneous left atrial appendage transcatheter occlusion (PLAATO system) to prevent stroke in high-risk patients with nonrheumatic atrial fibrillation: results from the international multi-center feasibility trials," Journal of the American College of Cardiology, vol. 46, no. 1, pp. 9-14, 2005.

[39] P. C. Block, S. Burstein, P. N. Casale et al., "Percutaneous left atrial appendage occlusion for patients in atrial fibrillation suboptimal for warfarin therapy: 5-year results of the PLAATO (Percutaneous Left Atrial Appendage Transcatheter Occlusion) Study," JACC Cardiovascular Interventions, vol. 2, no. 7, pp. 594-600, 2009.

[40] P. B. Sick, G. Schuler, K. E. Hauptmann et al., "Initial worldwide experience with the WATCHMAN left atrial appendage system for stroke prevention in atrial fibrillation," Journal of the American College of Cardiology, vol. 49, no. 13, pp. 14901495, 2007.

[41] R. B. Fountain, D. R. Holmes, K. Chandrasekaran et al., "The PROTECT AF (WATCHMAN left atrial appendage system for embolic PROTECTion in patients with atrial fibrillation) trial," American Heart Journal, vol. 151, no. 5, pp. 956-961, 2006.

[42] R. Virmani, F. D. Kolodgie, M. D. Dake et al., "Histopathologic evaluation of an expanded polytetrafluoroethylene- nitinol stent endoprosthesis in canine iliofemoral arteries," Journal of Vascular and Interventional Radiology, vol. 10, no. 4, pp. 445456, 1999.

[43] T. Nakai, E. P. Gerstenfeld, C. Stillson et al., "Intracardiac echocardiography guided implantation of a novel left atrial appendage occlusion device for preventing thrombembolism," Pacing and Cardiac Electrophysiology, vol. 24, p. 639, 2001.

[44] S. Rietbrock, E. Heeley, J. Plumb, and T. van Staa, "Chronic atrial fibrillation: incidence, prevalence, and prediction of stroke using the Congestive heart failure, Hypertension, Age $>75$, Diabetes mellitus, and prior Stroke or transient ischemic attack (CHADS2) risk stratification scheme," American Heart Journal, vol. 156, no. 1, pp. 57-64, 2008.

[45] D. R. Holmes, V. Y. Reddy, Z. G. Turi et al., "Percutaneous closure of the left atrial appendage versus warfarin therapy for prevention of stroke in patients with atrial fibrillation: a randomised non-inferiority trial," The Lancet, vol. 374, no. 9689, pp. 534-542, 2009.

[46] C. Van Walraven, R. G. Hart, D. E. Singer et al., "Oral anticoagulants vs aspirin in nonvalvular atrial fibrillation: an individual patient meta-analysis," Journal of the American Medical Association, vol. 288, no. 19, pp. 2441-2448, 2002.

[47] S. Kaul, E. Cingolani, M. Shehata, and G. A. Diamond, "How does left atrial appendage occlusion using the Watchaman device compare to dabibatran for reducing thromboembolic eventis in patients with nonvalvular atrial fibrillation," Journal of the American College of Cardiology, vol. 55, no. 10A, p. A4E39, 2010. 


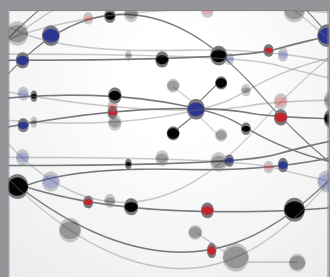

The Scientific World Journal
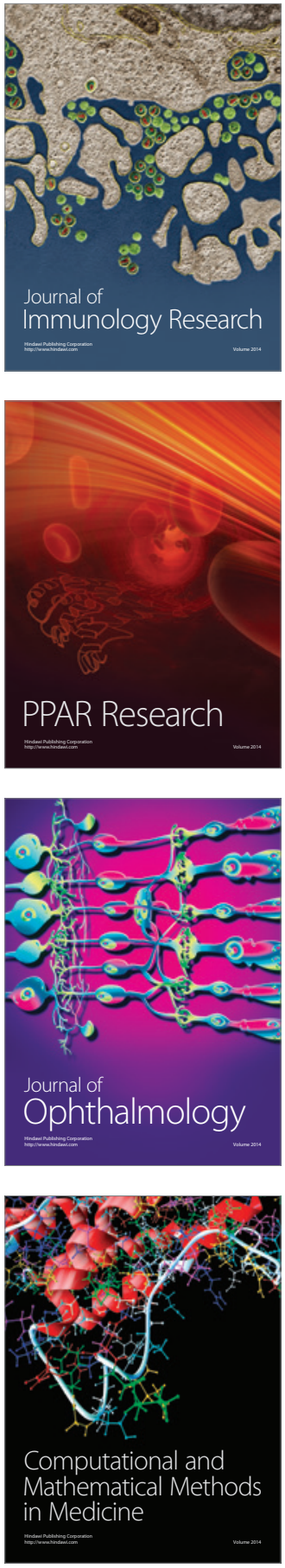

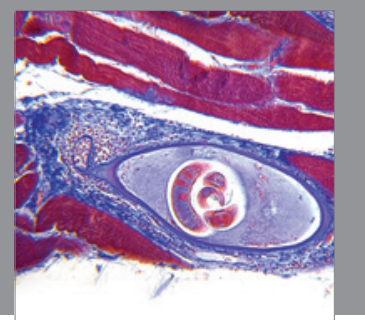

Gastroenterology

Research and Practice
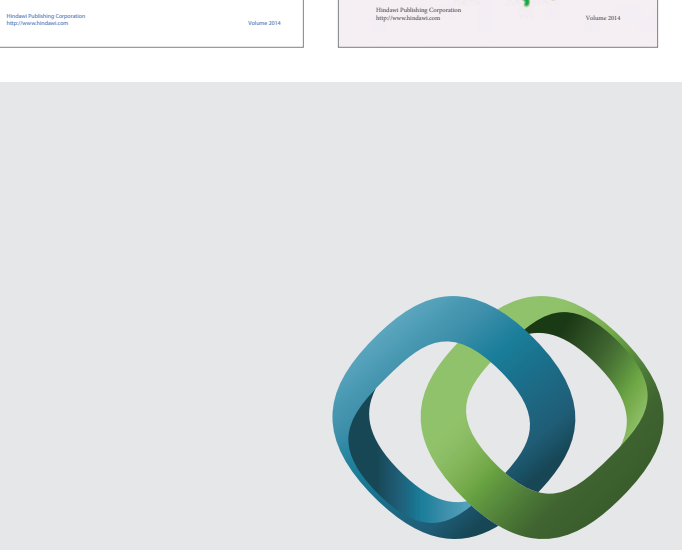

\section{Hindawi}

Submit your manuscripts at

http://www.hindawi.com
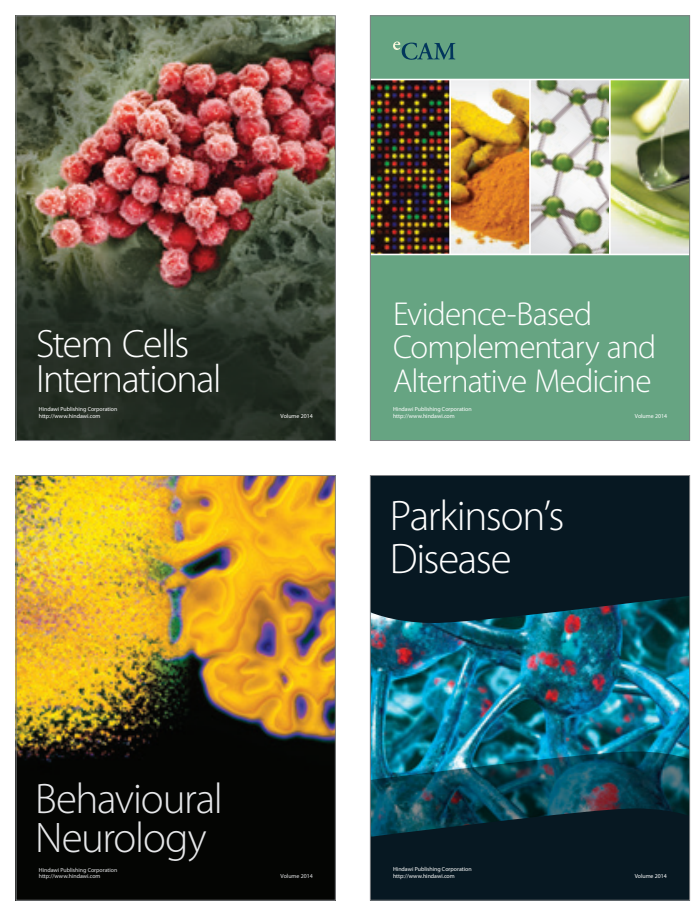

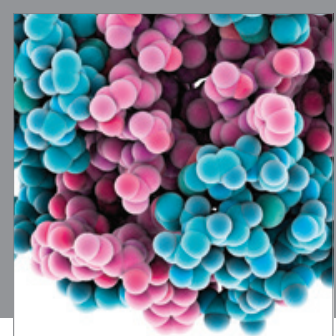

Journal of
Diabetes Research

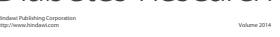

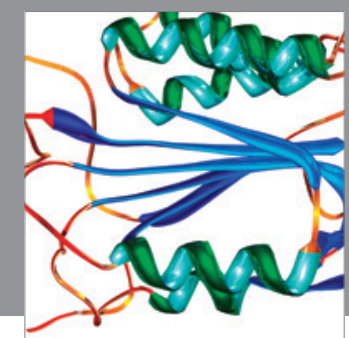

Disease Markers
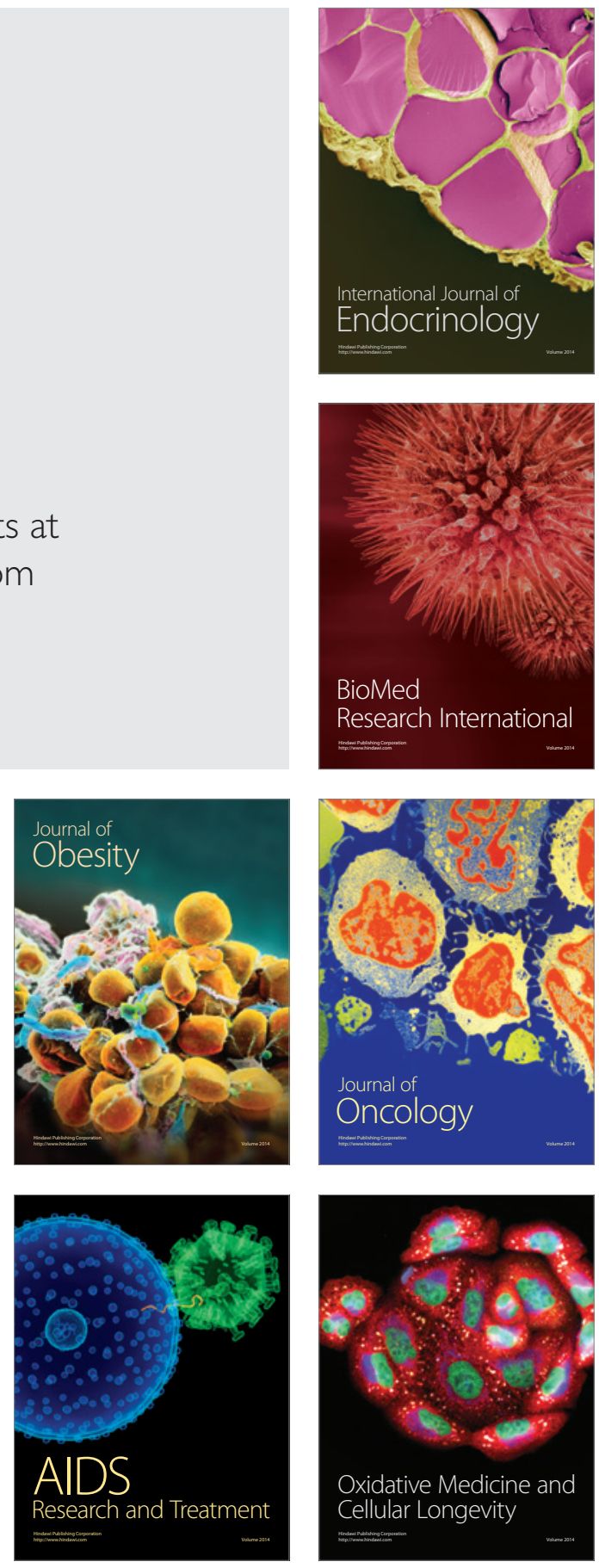